\title{
Software Resources for Remedial Physics Teaching in UK University Chemistry Departments
}

\begin{abstract}
Summary
There is a substantial minority of students who do not have the necessary physics background to tackle parts of most first year physical chemistry courses and for whom some kind of remedial action is required.

Software tools have been developed to address the problem with priority being assigned to topics most frequently occurring in the curricula. After discussion with colleagues, the topic of 'Particles and Waves in Chemistry' was selected. The tools consist of simulations (and high quality diagrams with relevant animations) designed to accompany tutorials or workshops. They are not 'stand-alone' programs and assume that the teacher has covered the basic groundwork. In this way, the material does not impose any learning style and should be treated as a simple resource similar to an illustration or photograph. Substantial numbers of numerical problems (in practice, an infinite number) are included for use as reinforcement learning tools - a technique that has been shown to be extremely effective in tackling specific numerical shortcomings.

Given that the overwhelming majority of the targeted students have chosen to avoid physics, great care has been taken to ensure that the context enclosing the materials is identifiable as chemistry only.
\end{abstract}

Subject area: Chemistry

\section{Description}

Software tools have been developed to help chemistry students with elementary problems in physics. These consist of simulations (and high quality diagrams with relevant animations) designed to accompany tutorials or workshops. They are not 'stand-alone' programs and assume that the teacher has covered the basic groundwork. Substantial numbers of numerical problems are included for use as reinforcement learning materials.

\section{Type of activity}

Simulation. Numerical practice.

\section{Content covered}

1. Electromagnetic waves.

2. Wavelength and frequency.

3. The electromagnetic spectrum.

4. Radiation energy.

5. Atomic spectroscopy.

6. The hydrogen atom spectrum.

7. Interference.

8. Diffraction.

9. De Broglie wavelength.

10. The photoelectric effect. 


\section{Application}

The tools have been designed to accompany tutorials or workshops together with a large number of numerical problems suitable for self-study. In addition the teacher may,

a. Specify the precision required.

b. Determine whether or not to insist on the correct precision being used.

c. Allow the students to control their own precision.

d. Determine the type of answer checking employed,

1. Level 1 answer checking responds by quoting the correct answer.

2. Level 2 answer checking responds with the accuracy - exact, within $1 \%$, within $5 \%$ and above $5 \%$.

Level 3 is more complex and gives a student three attempts at getting the answer right by responding with the accuracy figures used in level 2. Exact answers and those within 1\% are regarded as correct, whilst beyond $5 \%$ the student must try again. When level 3 checking is operative then the program keeps a record of student progress.

e. Completely replace the text with his/her own version.

\section{Further comments}

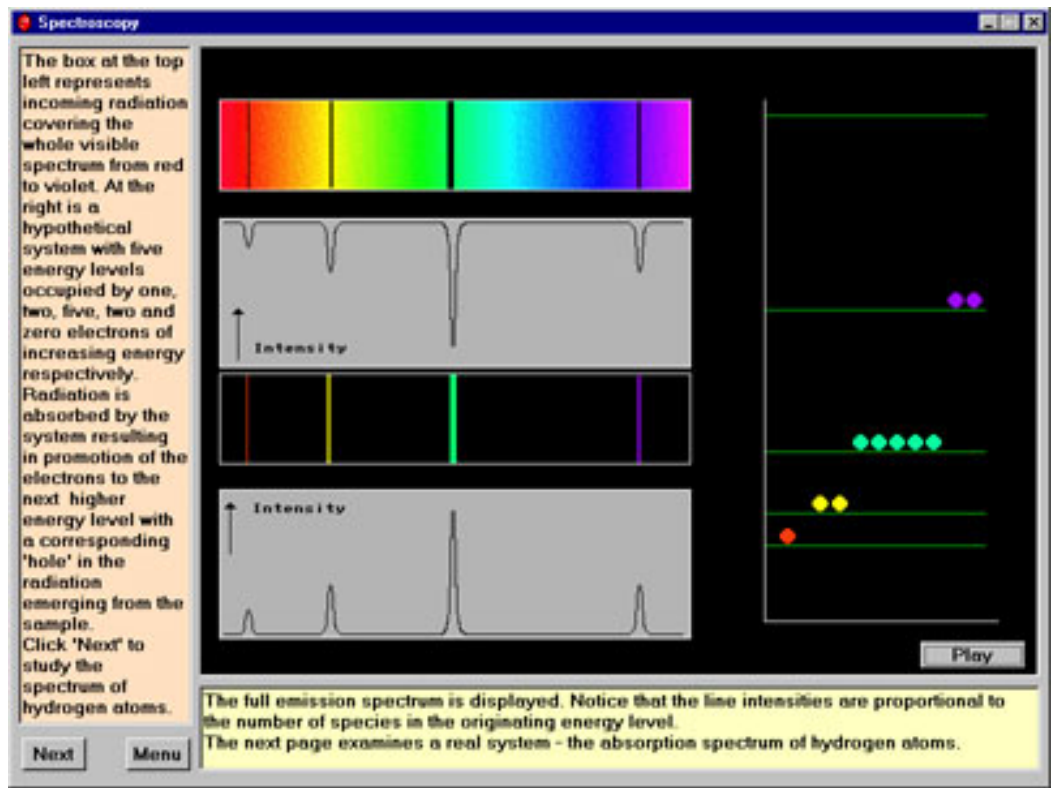

Figure 1 An animation illustrating the generation of atomic spectra by electronic excitation 


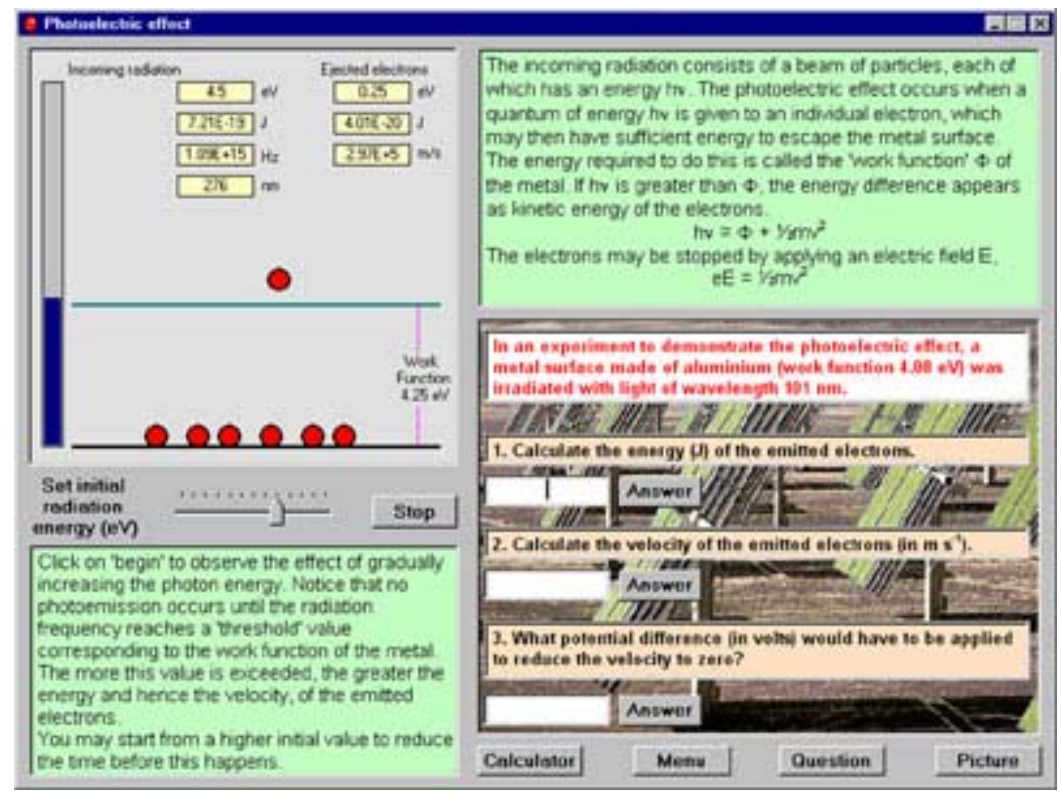

Figure 2 A simulation of the photoelectric effect illustrating the 'threshold energy'. A typical numeric problem has been requested.

\author{
Author(s) \\ Steve M. Walker \\ Department of Chemistry, University of Liverpool
}

\title{
Contact details
}

Steve M. Walker

Department of Chemistry, University of Liverpool

The Donnan Laboratories

Crown Street

University of Liverpool

Liverpool L69 7ZD

sk01@liverpool.ac.uk

\section{URL}

http://www.physsci.ltsn.ac.uk/devprojs/reports/phys_soft_res.htm

Development project: Software Resources for Remedial Physics Teaching in UK

University Chemistry Departments

http://www.physsci.ltsn.ac.uk/devprojs/Gcsephys.htm

Briefing paper: The Physics Problem

ftp://ftp.liv.ac.uk/FTPANON/ftpanon1/ftp/pub/chemistry/ltsn/physics

Link to software cited in article 